\title{
Heterogeneity in the Nitroxide Micro-Environment: Polarity and Proticity Effects in Spin-Labeled Proteins Studied by Multi-Frequency EPR
}

\author{
E. Bordignon • H. Brutlach • L. Urban • K. Hideg • \\ A. Savitsky • A. Schnegg • P. Gast • M. Engelhard • \\ E. J. J. Groenen · K. Möbius • Heinz-Juergen Steinhoff
}

Received: 12 June 2009/Published online: 12 November 2009

(C) The Author(s) 2009. This article is published with open access at Springerlink.com

\begin{abstract}
This study aims to investigate the $g_{x x}$ heterogeneity of the $g$-tensor commonly observed in high-field electron paramagnetic resonance (EPR) spectra of nitroxide spin-labeled sites in proteins. This heterogeneity is addressed in terms of spin-label populations characterized by different polarity and H-bonding properties of the nitroxide micro-environment. The $g_{x x}$ value for each population is determined from the fit of continuous-wave high-field spectra obtained at 95, 275 and $360 \mathrm{GHz}$ with a series of nitroxide spin-labels covalently attached to different sites in both membrane and water-soluble proteins. The spin-labeled proteins investigated include
\end{abstract}

\footnotetext{
E. Bordignon

Laboratory for Physical Chemistry, ETH Zurich, Wolfgang-Pauli-Str. 10, 8093 Zurich, Switzerland

H. Brutlach · L. Urban · H.-J. Steinhoff $(\bowtie)$

Department of Physics, University of Osnabrück, Barbarastrasse 7, 49076 Osnabrück, Germany e-mail: hsteinho@uos.de

K. Hideg

Institute of Organic and Medicinal Chemistry, University of Pécs, PO Box 99, 7602 Pecs, Hungary
}

A. Savitsky

Max-Planck Institute for Bioinorganic Chemistry, Stiftstrasse 34-36, 45470 Mülheim/Ruhr, Germany

\author{
A. Savitsky · A. Schnegg $\cdot$ K. Möbius \\ Department of Physics, Free University of Berlin, Arnimallee 14, 14195 Berlin, Germany
}

\author{
A. Schnegg \\ Department of Si-Photovoltaics, Helmholtz-Centre Berlin for Materials and Energy, \\ Kekuléstrasse 5, 12489 Berlin, Germany \\ P. Gast · E. J. J. Groenen \\ Huygens Laboratory, Leiden University, Niels Bohrweg 2, 2333 CA Leiden, The Netherlands \\ M. Engelhard \\ Max-Planck Institute for Molecular Physiology, Otto-Hahn-Strasse 11, 44227 Dortmund, Germany
}


sensory rhodopsin II and its cognate transducer molecule (HtrII) from Natronomonas pharaonis both in micelles and membranes, bacteriorhodopsin from Halobacterium salinarum in native purple membrane lipid bilayers and water-soluble colicin A from Escherichia coli. To avoid contributions to the $g_{x x}$ spectral features of the nitroxide label due to nuclear quadrupole interactions arising from ${ }^{14} \mathrm{~N}$ nuclei, and to simplify the nitrogen hyperfine pattern, methanethiosulfonate spin labels, containing the ${ }^{15} \mathrm{~N}$ isotope $(I=1 / 2)$ in some experiments, were employed. A consistent analysis of all multi-frequency EPR spectra revealed three distinct $g_{x x}$ values, $g_{x x}^{i}$, for each investigated position of the labeled proteins. In contrast, distinctly different nitrogen hyperfine splittings $A_{z z}$ of the nitroxides in the various labeled proteins could not be resolved, but rather an average hyperfine splitting $\bar{A}_{z z}$ was obtained. The $g_{x x}^{i}$ values as well as the fractions of the different nitroxide populations were found to be correlated with the average hyperfine constant $\bar{A}_{z z}$, a parameter which likewise is known to be sensitive to the local polarity of the spin-label micro-environment. Plotting the different $g_{x x}^{i}$ values obtained for each EPR spectrum versus $\bar{A}_{z z}$ of the labeled proteins reveals new interesting aspects of the nitroxide label micro-environment in terms of polarity and $\mathrm{H}$-bonding propensity (proticity). Linear approximations of the different regions of the plot $g_{x x}^{i}$ versus $\bar{A}_{z z}$ are presented and compared with theoretical and experimental data available from the literature.

\section{Introduction}

In site-directed spin-labeling, paramagnetic molecules (typically nitroxide radicals) are specifically bound to engineered cysteine residues of the protein under investigation. A detailed characterization of the protein structure and topology by electron paramagnetic resonance (EPR) techniques can be obtained (1) from the spin-label dynamics that is correlated to protein secondary and tertiary structures, (2) from the accessibility of the nitroxide toward water and lipid phases, and (3) from interspin distances between specifically engineered doubly labeled mutants (for a recent review, see Ref. [1]). Additionally, the local polarity and proticity (defined as the propensity to form H-bonds) of the nitroxide micro-environment can be detected with high sensitivity.

The micro-environment polarity exerts its influence primarily on the $g$-tensor parameter $g_{x x}$ and nitrogen hyperfine tensor parameter $A_{z z}$ due to the electric field produced along the $x$-axis of the nitroxide ( $\mathrm{N}-\mathrm{O}$ bond direction) by specific protein side chains and water molecules surrounding the spin-label side chain. The microenvironment proticity mainly affects the nitroxide $g$-tensor and becomes observable, at sufficiently high magnetic field beyond that of X-band EPR $(9.5 \mathrm{GHz})$, predominantly as a change in the $g_{x x}$ value [2].

Several cases have been reported where the polarity/proticity characteristics of the EPR parameters measured in frozen samples were a good description of the protein or lipid bilayer situations at physiological temperature [3-7]. Deviations might occur when (slow) freezing of the sample modifies an existing conformational equilibrium or disrupts the hydrogen bond network around the spin label. Disentangling polarity from proticity effects is often possible by determining concomitantly both $A_{z z}$ and $g_{x x}$. The component $A_{z z}$ has been shown to be a sensitive 
parameter for electric field effects due to shifts in the spin-density from oxygen toward the nitrogen atom, depending on the electric field strength along the $\mathrm{N}-\mathrm{O}$ bond [2]. The $A_{z z}$ parameter can be obtained by analysis of the EPR spectra detected at temperatures below $200 \mathrm{~K}$ already at X-band frequencies. To resolve the anisotropy of the $g$-tensor, i.e., to determine $g_{x x}$, a spectral resolution higher than that achieved by X-band EPR is required. For nitroxide labels, the $g$ anisotropy is already resolved at W-band EPR $(95 \mathrm{GHz} / 3.4 \mathrm{~T})$ and can be further exploited at higher frequency/field settings (e.g., $275 \mathrm{GHz} / 9.8 \mathrm{~T}$ and $360 \mathrm{GHz} / 12.9 \mathrm{~T}$ ). Theoretical studies have been performed by several groups to describe the effect of polarity and propensity for $\mathrm{H}$-bond formation on the $A$ - and $g$-tensor elements at different levels of sophistication and with different focuses [2, 8-11].

Distinguishing between polarity and proticity effects is usually performed by measuring $g_{x x}$ and $A_{z z}$ in frozen samples of several site-specifically nitroxidelabeled, molecular systems. The linear plot of $g_{x x}$ versus $A_{z z}$ exhibits different slopes for polar and protic micro-environments of the nitroxides and, thus, enables to disentangle, for each labeled site, polarity effects and the availability of H-bonds [2, 7]. Such an analysis can be complicated by the presence of multiple $g_{x x}$ components in the spectrum from different complexes or by " $g_{x x}$ strain" effects from the environment [12], leading to detectable shoulders in the $g_{x x}$ spectral region or to a broadening of the EPR lines, respectively.

The composite nature of the $g_{x x}$ region of the high-field EPR spectra was recognized earlier, for example, in spin-labeled lipids at $95 \mathrm{GHz}$ [13] and in spinlabeled azurin proteins at $275 \mathrm{GHz}$ [14]. The authors of these studies suggested that two nitroxide populations are being observed that are characterized by the presence or absence of a H-bond to the nitroxide moiety. A high-field electron-nuclear double resonance study [13] confirmed the presence of one $\mathrm{H}$-bond in the water-surrounded radical component with lower $g_{x x}$, and gave the orientation of the water molecule with respect to the nitroxide plane. The polarity/proticity investigation, performed at $95 \mathrm{GHz}$ on the spin-labeled transducer, HtrII, in the sensory rhodopsin II (SRII)/HtrII complex reconstituted in the membrane [5], showed an even more complex $g_{x x}$ region with distinct shoulders which could not be fitted using only two $g_{x x}$ components. To investigate the origin of these complex spectral features, we analyzed in detail the heterogeneity in the $g_{x x}$ region of a collection of EPR spectra detected at 95, 275 and $360 \mathrm{GHz}$. The multi-frequency spectral fits performed for various protein sites with different spin-label moieties revealed the reproducible existence of three different spectral components characterized by $g_{x x}$ values separated by $\approx 4 \times 10^{-4}$. Notably, this shift corresponds to the theoretical value expected for the addition of one H-bond to the NO group. The correlation observed between the $g_{x x}$ values, as well as the fractions of each component population, versus $A_{z z}$ will be discussed, and the origin of the three populations will be addressed.

\section{Materials and Methods}

The $95 \mathrm{GHz} / 3.4 \mathrm{~T}$ continuous-wave (cw) EPR measurements presented in this work were performed on a home-built W-band EPR spectrometer equipped with a $\mathrm{TE}_{011}$ 
cavity whose setup was described elsewhere [5]. The sample suspensions were filled into quartz capillaries with inner diameter of $0.6 \mathrm{~mm}$ and investigated at $175 \mathrm{~K}$ with protein concentrations in the $200-400 \mu \mathrm{M}$ range. The microwave power was attenuated to $3 \mu \mathrm{W}$. Magnetic field modulation frequency was $100 \mathrm{kHz}$ and the amplitude $0.15 \mathrm{mT}$.

The presented W-band spectra are averages of two to six measurements. The microwave phase was adjusted to an estimated accuracy of $\pm 1^{\circ}$. Calibration of the magnetic field setting was performed daily using a $\mathrm{Mn}^{2+} / \mathrm{MgO}$ standard as described in Ref. [15]. Repeated test calibrations showed that the $B_{0}$ field value can be determined with a mean deviation of $<0.02 \mathrm{mT}$. The $275 \mathrm{GHz}$ cw EPR experiments were performed at $160 \mathrm{~K}$ on a home-built EPR spectrometer described elsewhere [16]. The frequency of the field modulation was $1.3 \mathrm{kHz}$; the modulation amplitude was $0.15 \mathrm{mT}$. The magnetic field was calibrated using as a reference the constant $g_{z z}$ value of 2.0022 for the present spin-labeled protein spectra.

The $360 \mathrm{GHz}$ cw EPR experiments were performed with a home-built superheterodyne quasi-optical spectrometer, consisting of a 14-T superconducting magnet and a 360-GHz heterodyne microwave board, as described elsewhere [17]. Spectra were recorded without microwave resonator in induction mode with a small amount (about $20 \mu \mathrm{l}$ ) of sample placed onto a sample holder. The probe head was then introduced into the precooled static helium contact cryostat inside the superconducting magnet. All experiments were performed at $140 \mathrm{~K}$. The six-line EPR spectrum of a $\mathrm{Mn}^{2+} / \mathrm{MgO}$ standard [15] in a polystyrene matrix (to prevent mixing with the protein sample), placed near the sample, was recorded simultaneously for $g$-factor calibration. Subsequently, it was subtracted from the EPR spectrum of the sample in order to obtain an undistorted spectrum.

The simulated powder spectra were fitted to the experimental spectra using a modified version of the program DIPFIT [18], in which up to three different $g_{x x}$ values and the corresponding population fractions (fr) were fitted simultaneously. To reduce the number of fit parameters, the line width broadening function was determined from the $g_{y y}$ and $g_{z z}$ spectral regions. The homogeneous line width was determined from the $g_{z z}$ region, since it is constant in good approximation [8], while the inhomogeneous line width was taken from the $g_{y y}$ region. The $A_{x x}$ and $A_{y y}$ components of the hyperfine coupling tensor, which are less sensitive to polarity changes, were approximated as 0.52 and $0.45 \mathrm{mT}$ for ${ }^{14} \mathrm{~N}$-isotope spin-label side chains, and 0.72 and $0.64 \mathrm{mT}$ for ${ }^{15} \mathrm{~N}$-isotope spin-label side chains.

The ${ }^{14} \mathrm{~N}$ spin-label (1-oxyl-2,2,5,5,-tetramethyl-3-pyrroline-3-methyl)methanethiosulfonate (MTSSL, in the following named as R1) [19] used in the present study has been purchased from Toronto Research Chemicals and used without further purification. All other spin labels were synthesized by one of the authors (K.H.): (1-oxyl-2,2,5,5,-tetramethyl-3-pyrroline-3-methyl)methanethiosulfonate ${ }^{15} \mathrm{~N}-\mathrm{D} 15\left({ }^{15} \mathrm{~N}-\mathrm{MTS}\right.$, in the following named as R2) and 1-oxyl-2,2,5,5,-tetramethyl, 2,5-dihydro-1H-pyrrol-3-carboxylic acid (2-methanethiosulphonyl-ethyl) amide (in the following named as R3). The solvents used were bidistilled water $\left(\varepsilon_{293 \mathrm{~K}}=80.4\right)$, ortho-terphenyl $\left(\varepsilon_{298 \mathrm{~K}}=2.8\right)$ and dimethyl sulfoxide (DMSO) $\left(\varepsilon_{293 \mathrm{~K}}=48\right)$. All solvents were purchased from Sigma-Aldrich. The spin-labeled proteins were prepared as described elsewhere [20-22]. 


\section{Results}

This investigation focuses on the interpretation of the heterogeneity observed in the $g_{x x}$ region detected in the low-temperature W-band EPR spectra of spin-labeled membrane and water-soluble proteins. Figure 1 shows a selection of EPR spectra with typical signal-to-noise ratio of spin-labeled colicin A (ColA) in water, and of spin-labeled SRII in the presence or absence of its cognate transducer HtrII reconstituted in purple membrane lipids. Most spectra show two or three pronounced shoulders in the $g_{x x}$ region together with the well-known overall displacement toward lower or higher $B_{0}$ fields, according to the respective changes in polarity. This is accompanied by $A_{z z}$ shifts in the high-field region. The fit of the spectra with only one set of $g_{x x}, g_{y y}, g_{z z}$ values was found to be unsatisfactory, especially in the $g_{x x}$ spectral region. The fits shown in Fig. 1 (gray lines) well reproduce the features observed in the low-field region. They were obtained by a superposition of three spectra different only in the $g_{x x}$ values (see Table 1). Three distinct $g_{x x}$ values were needed to convincingly fit the low-field region of the spectra. The high-field region, however, could be fitted with a unique $\bar{A}_{z z}$ parameter, representing the average nitroxide hyperfine splitting for each investigated position of the labeled protein with its site-specific local polarity of the micro-environment. This suggests that even if different $A_{z z}$ might be associated with each $g_{x x}$ component, their presence in the spectrum is not detectable due to the large inhomogeneous EPR line width.

The additional presence of three partially unresolved $A_{x x}$ triplet splittings due to the hyperfine coupling with ${ }^{14} \mathrm{~N}$ complicates the analysis of the features in the $g_{x x}$ region even more. The decrease in the root-mean-square deviation in the fitting procedure when going from two to three $g_{x x}$ components did not always allow an unambiguous assignment of the number of components in the spectrum. Thus, to avoid the effects of the $A_{x x}$ hyperfine lines and of the ${ }^{14} \mathrm{~N}$ nuclear quadrupole interaction [10] on the observed shoulders in the $g_{x x}$ region, W-band experiments on ${ }^{15} \mathrm{~N}$-substituted spin labels were performed on the SRII-V17 protein system (Fig. 2). Due to the reduction of the inhomogeneous line width by perdeuteration, the nitrogen hyperfine splitting (doublets for $\mathrm{R} 2, I=1 / 2$, instead of triplets for $\mathrm{R} 1$, $I=1$ ) is clearly observed in all $g$ regions (Fig. 2). Moreover, the absence of nuclear quadrupole interaction further helped to discern the pattern of shoulders and clarify the assignment of the nitroxide populations.

Interestingly, the same shoulders seen in the case of the ${ }^{14} \mathrm{~N}$ isotope are still distinguishable in the $g_{x x}$ region of the spectrum recorded with the R 2 spin label. For comparison, the spectra of the R2 spin label alone dissolved in ortho-terphenyl (apolar aprotic solvent) and deuterated DMSO (polar aprotic solvent), for which only one $g_{x x}$ component is present, are also shown in Fig. 2.

Figure 3 highlights the $g_{x x}$ region of the ${ }^{15} \mathrm{~N}$-containing SRII-V17R2 and the spectral fits performed with one, two or three $g_{x x}$ components. In accordance with the results obtained with the ${ }^{14} \mathrm{~N}$ isotope, the three-component fit led to the smallest deviation from the experimental line shape, and only the fit with three components represents the three pronounced shoulders of the experimental line. This confirms the presence of three nitroxide populations in the sample investigated. The resulting 


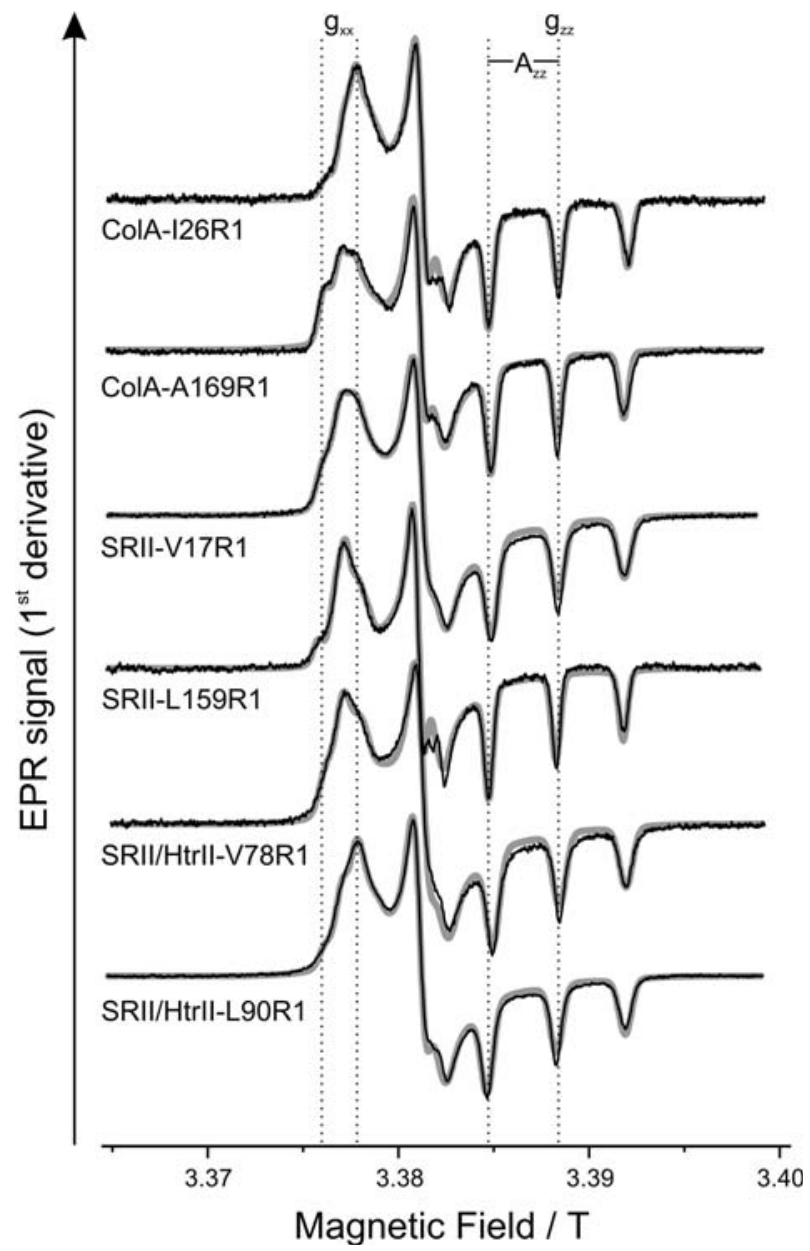

Fig. 1 Rigid-limit W-band EPR (95 GHz) spectra (black lines, $T=175 \mathrm{~K}$ ) of selected spin-labeled mutants of colicin A (ColA) from E. coli in aqueous solution, sensory rhodopsin II (SRII) from $N$. pharaonis and its cognate transducer molecule (HtrII) reconstituted in purple membrane lipids. The spin-label used was MTSSL (R1 side chain) with the ${ }^{14} \mathrm{~N}$ isotope. The spectra show the presence of shoulders in the $g_{x x}$ region (low-field region) for many of the investigated positions. Vertical bars in the $g_{x x}$ and $A_{z z}$ regions guide the eye to better visualize the different shoulders and the change in $A_{z z}$. The gray lines represent the best fits of simulated spectra with three different $g_{x x}^{i}$ values to the experimental ones

$g_{x x}$ values are given in Table 1, together with all parameter values obtained from the spectra presented in Figs. 1 and 2.

The three nitroxide populations were also investigated at higher $B_{0}$ field to increase spectral resolution. In Fig. 4 two spectra detected at $95 \mathrm{GHz}$ are compared to those recorded at 275 and $360 \mathrm{GHz}$ for the selected protein sites SRII-V17R1 and SRII/HtrII-L93R1, respectively. The increased spectral resolution unequivocally reveals the presence of three different components. Superimposed to the experimental spectra, the best fits obtained with three $g_{x x}$ components are presented (see 


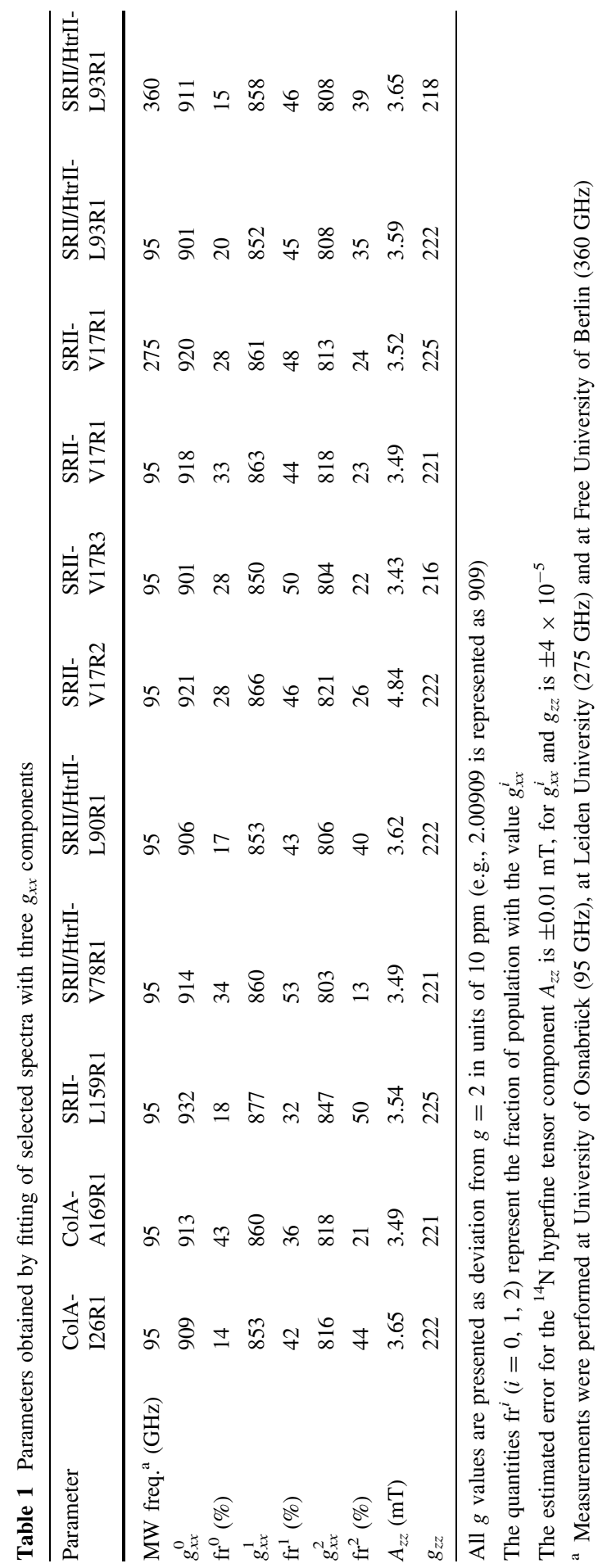




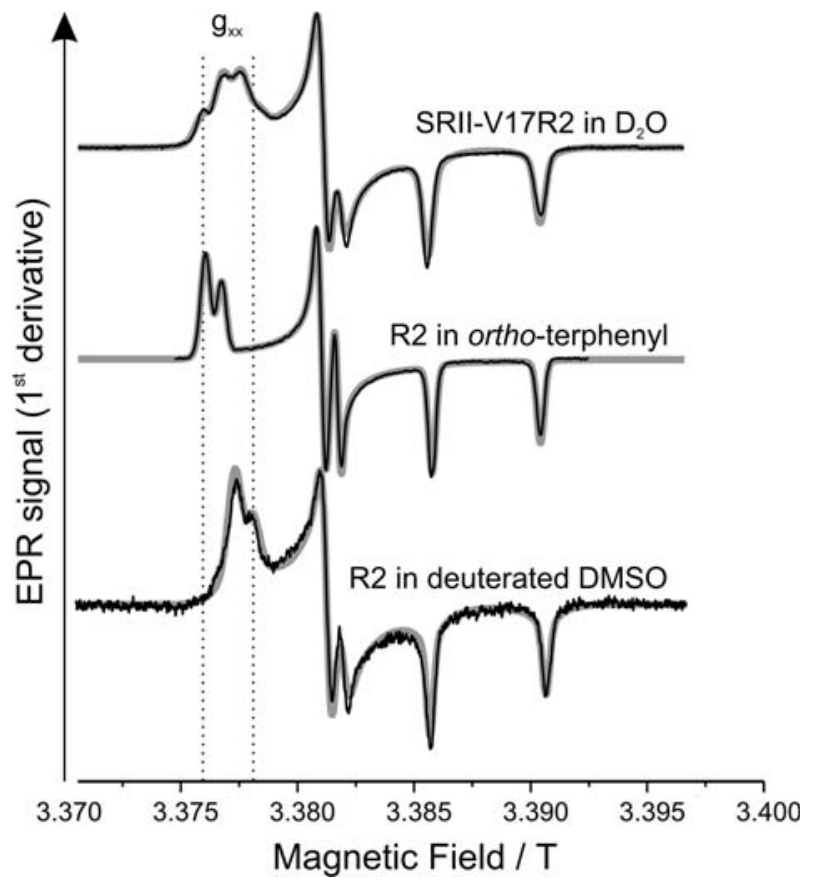

Fig. 2 Rigid-limit W-band spectrum of SRII-V17R2 showing distinct shoulders in the $g_{x x}$ region. The spin-label used was perdeuterated ${ }^{15} \mathrm{~N}$-MTS (R2) in $\mathrm{D}_{2} \mathrm{O}$ buffer. For comparison, the spectra of R2 dissolved in ortho-terphenyl [4] and deuterated DMSO, which present only one $g_{x x}$ component, are also shown. The doublet visible in the $g_{x x}$ region for R2 dissolved in different solvents is due to the hyperfine splitting $A_{x x}$

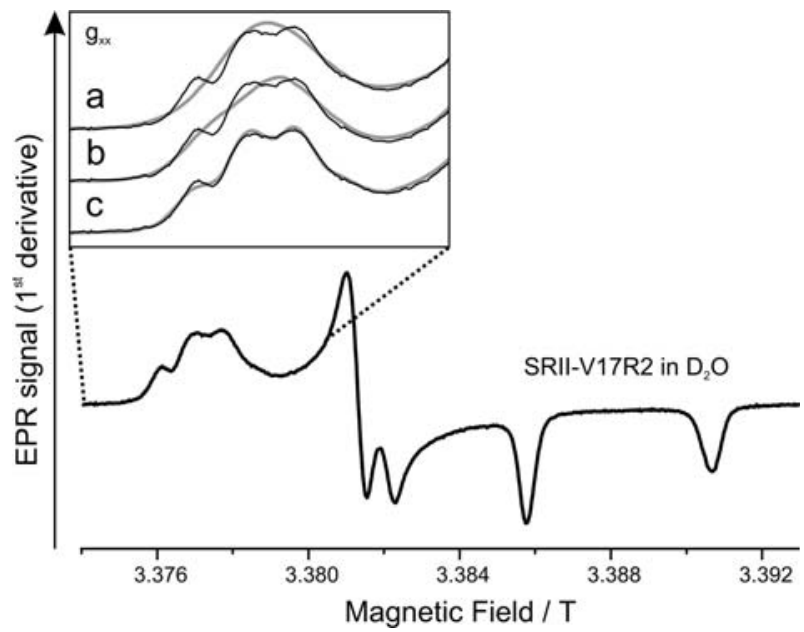

Fig. 3 Rigid-limit W-band spectrum of SRII-V17 spin labeled with perdeuterated ${ }^{15} \mathrm{~N}-\mathrm{MTS}$ (R2) in $\mathrm{D}_{2} \mathrm{O}$ buffer. $a$ The $g_{x x}$ region of the spectrum is highlighted. The gray line corresponds to a spectral fit with one $g_{x x}$ value. $b$ Fit with two different $g_{x x}$ values. $c$ Fit with three different $g_{x x}$ values 

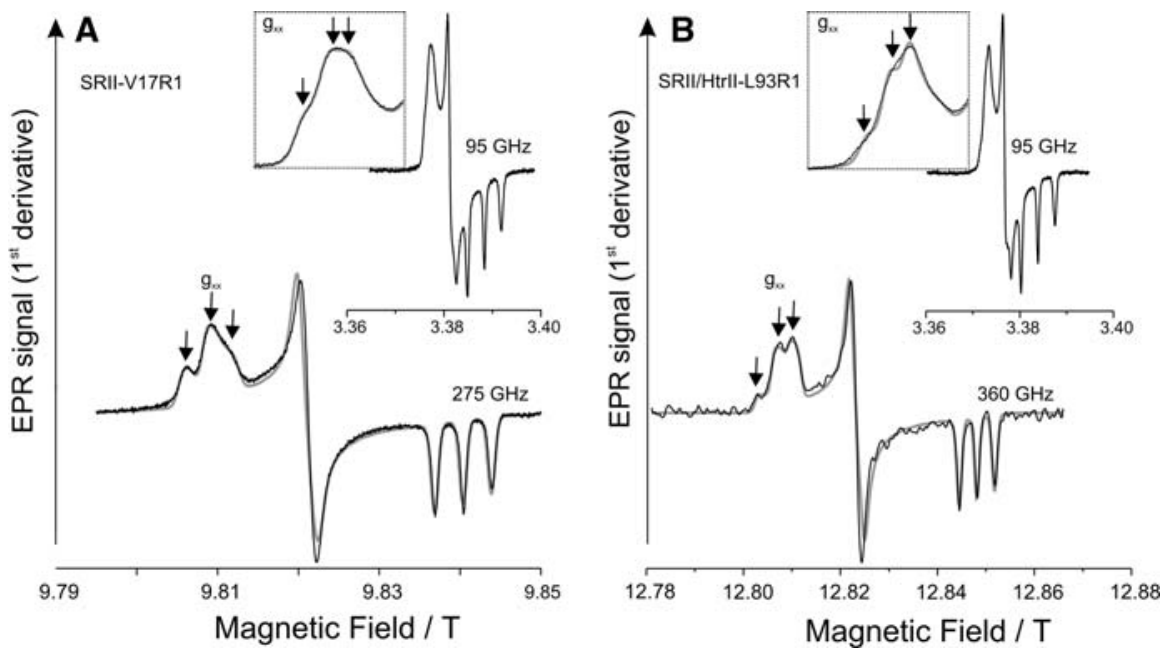

Fig. 4 Rigid-limit experimental high-field EPR spectra for SRII-V17R1 and SRII/HtrII-93R1 recorded at different microwave frequencies and corresponding magnetic fields. Black lines represent the experimental data, and gray lines correspond to the fitting of the simulated spectra to the experimental data using three different $g_{x x}$ values (vertical arrows). Insets at the top show the amplified low-field region $\left(g_{x x}\right)$ of the $95 \mathrm{GHz}$ spectra and the spectral fits. a Spectra of SRII-V17R1 recorded at $95 \mathrm{GHz}$ (upper panel, $175 \mathrm{~K}$ ) and $275 \mathrm{GHz}$ (lower panel, $70 \mathrm{~K}$ ). b EPR spectra of SRII/HtrII-L93R1 recorded at $95 \mathrm{GHz}$ (upper panel, $175 \mathrm{~K}$ ) and $360 \mathrm{GHz}$ (lower panel, $160 \mathrm{~K}$ )

Table 1). Both the fraction of each component and the $g_{x x}$ values required for the best fit are found to coincide within experimental error at all microwave (MW) frequencies. This further confirms that the shoulders observed in the EPR spectra at W-band do indeed arise from three different nitroxide populations, and that the fits of W-band spectra give reliable information on the nitroxide populations.

In Fig. 5a the three $g_{x x}$ values $\left(g_{x x}^{i}, i=0,1,2\right)$ are plotted versus the average values $\bar{A}_{z z}$ and superimposed to the previously published plot [2]. Interestingly, the $g_{x x}^{i}$ values obtained from the spectral fits fall into three distinct regions of the plot and show a correlation with the $\bar{A}_{z z}$ values. The slope of each linear regression indicates the change in $g_{x x}$ due to the polarity changes, which is linearly dependent on the change in $A_{z z}$. The main difference with respect to the previously published $g_{x x}$ versus $A_{z z}$ plot [2] lies in the fact that there is an almost constant gap of $\approx 4 \times 10^{-4}$ separating $g_{x x}^{i}$ from $g_{x x}^{i+1}$, independently of the $\bar{A}_{z z}$ values. Similar slopes are found for the linear regressions of each $g_{x x}$ component versus $\bar{A}_{z z}$.

The plot in Fig. $5 \mathrm{~b}$ represents the variation of the fraction $\mathrm{fr}^{i}$ of each $g_{x x}^{i}$ component with respect to the average polarity of the environment (described by $\left.\bar{A}_{z z}\right)$. Interestingly, in the polarity region investigated $\left(\bar{A}_{z z}\right.$ ranging from 3.4 to $3.7 \mathrm{mT})$, the fraction $\mathrm{fr}^{1}$ of the intermediate $g_{x x}^{1}$ remains almost constant $(\approx 50 \%$ of the overall population), while the fraction $\mathrm{fr}^{0}$ of $g_{x x}^{0}$ decreases almost to zero by increasing the polarity $\left(\bar{A}_{z z}\right.$ increasing from 3.4 to $\left.3.7 \mathrm{mT}\right)$, and, on the contrary, the fraction $\mathrm{fr}^{2}$ of $g_{x x}^{2}$ increases. The analysis of the "fraction plot" indicates that the $g_{x x}^{0}$ component is prevalently populated in apolar environments, while the $g_{x x}^{2}$ component describes a fraction of population energetically favored in polar 


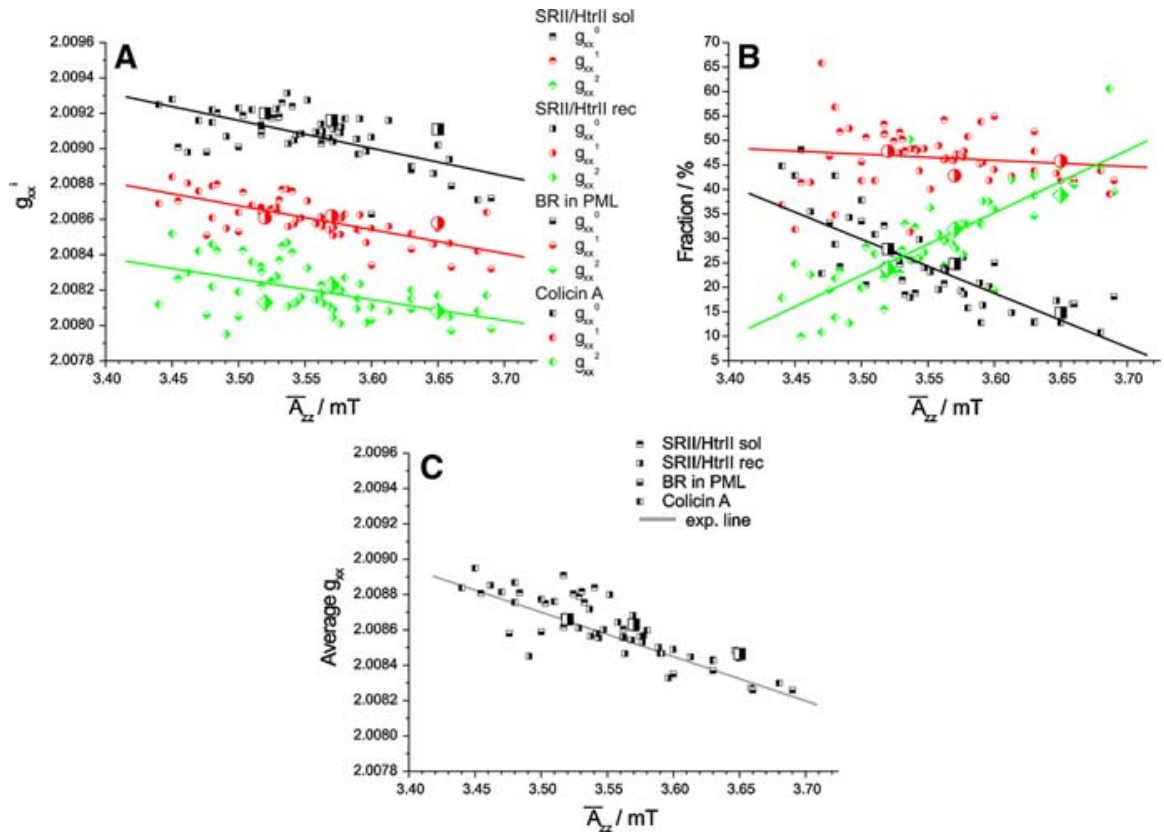

Fig. 5 a Plot of $g_{x x}$ versus $\bar{A}_{z z}$ obtained by fitting the W-band EPR spectra of a series of spin-labeled positions in different proteins. The three spectral components $\left(g_{x x}^{0}, g_{x x}^{1}, g_{x x}^{2}\right)$ are shown as black, green and red symbols, respectively. Each symbol denotes the protein investigated (depicted in the figure). The large symbols correspond to data obtained from the fits of the 275 and $360 \mathrm{GHz}$ spectra. b Plot of the fraction $\mathrm{fr}^{i}$ of each $g_{x x}^{i}$ component versus $\bar{A}_{z z}$. Color code is as in a. c Plot of the average $g_{x x}$ values (calculated as $\left.g_{x x}^{0} \cdot \mathrm{fr}^{0}+g_{x x}^{1} \cdot \mathrm{fr}^{1}+g_{x x}^{2} \cdot \mathrm{fr}^{2}\right)$ versus $\bar{A}_{z z}$. The gray line corresponds to the experimental protic line published previously [5]

environments. The plot of the average value of $g_{x x}$, as calculated by using the obtained fractions, is presented in Fig. 5c. The comparison with the previously obtained data based on spin labels attached to a multitude of protein sites and dissolved in solvents [5] shows good agreement.

\section{Discussion}

The multi-frequency $(95,275,360 \mathrm{GHz})$ high-field EPR approach used in this work allows to unveil the invariant presence of three different nitroxide populations in a series of spin-labeled sites in a variety of proteins. The use of ${ }^{14} \mathrm{~N}$ and ${ }^{15} \mathrm{~N}$ isotopes in the nitroxides unambiguously confirms the assignment of a distinct $g_{x x}$ value to each population. This assignment is based on the fitting of simulated cw EPR spectra to the experimental ones and shows that effects of nitrogen hyperfine or quadrupole interactions do not interfere with the conclusions drawn. The origin of the three populations observed will be discussed in the following.

The fact that the slopes $\left(1.2-1.6 \times 10^{-3} \mathrm{mT}^{-1}\right)$ of the obtained straight lines $g_{x x}^{i}$ versus $\bar{A}_{z z}$ in Fig. 5 are equal within experimental errors indicates that each $g_{x x}$ 
component correlates independently with the (averaged) local polarity. Thus, the slopes of these lines reflect exclusively the influence of polarity changes. These slope values obtained are in good agreement with the theoretical prediction of $5.1 \times 10^{-3} \mathrm{mT}^{-1}$ [2], which, however, was shown to be quite sensitive to differences in the angle $\varphi$ between the $\mathrm{N}-\mathrm{O}$ bond and the plane of the attached fivemembered ring. In fact, for $\varphi=20^{\circ}$, a slope of $1.1 \times 10^{-3} \mathrm{mT}^{-1}$ was calculated. Furthermore, a slope of $0.8 \times 10^{-3} \mathrm{mT}^{-1}$ was obtained from measurements of unbound MTSSL in different solvents [8].

On the other hand, the vertical shifts between the three regression lines in the $g_{x x}^{i}$ versus $\bar{A}_{z z}$ plot of Fig. 5 can be regarded as representing additional effects due to $\mathrm{H}$-bonds to the nitroxide label. Starting from the fact that the analysis of the fraction plot indicates that the $g_{x x}^{0}$ component is prevalently populated in apolar environments, while the $g_{x x}^{2}$ component describes a fraction of population energetically favored in polar environments, one might surmise that $g_{x x}^{0}$ corresponds to a non-Hbonded nitroxide moiety and $g_{x x}^{2}$ to a H-bonded moiety, mostly favored in polar environments, which are usually also characterized by a higher amount of bulk water molecules. If this was the case, the difference between the average values of $g_{x x}^{0}$ and $g_{x x}^{2}$ of almost $900 \mathrm{ppm}$ would not be in line with both the theoretical $\Delta g_{x x}$ value of $\approx 400 \mathrm{ppm}$, as calculated for the addition of one $\mathrm{H}$-bond [2, 8], and the experimental $\Delta g_{x x}$ value of $600 \mathrm{ppm}$, as found in the case of the spin-labeled 5-doxyl stearic acid in lipid membranes [6]. The $\Delta g_{x x}$ of $2100 \mathrm{ppm}$ calculated by Engström et al. [11] for the addition of one H-bond is admittedly exceeding the experimentally observed values by far.

As a hypothesis, the presence of a constantly populated third fraction characterized by $g_{x x}^{1}$ could be possibly explained by (1) a different H-bond characterized by a different length to the nitroxide, (2) the presence of a H-bonded spin-label rotamer with a different nitroxide plane orientation, (3) the effect of the bulk polarity of the water in the absence of a specific H-bond. However, one has to keep in mind that (i) $g_{x x}^{1}$ is not species-specific, (ii) it is present in almost $50 \%$ of the nitroxide population throughout the polarity range investigated, and (iii) it is not affected by the use of a different spin-label side chain, namely R3 (see Table 1). Hence, these observations would pose serious problems to validate such a hypothesis.

A second possible explanation is to surmise that $g_{x x}^{2}$ characterizes the nitroxide population having two H-bonds, and $g_{x x}^{1}$ the population with one H-bond. From Fig. 5 it is evident that only a micro-environment characterized by high polarity $\left(\bar{A}_{z z}=3.7 \mathrm{mT}\right)$ can favor the formation of a second H-bond to the nitroxide, which otherwise is not formed at appreciable amount $\left(<20 \%\right.$ for $\left.\bar{A}_{z z}<3.5 \mathrm{mT}\right)$. Calculation of $g_{x x}$ and $A_{z z}$ values in the presence of one and two H-bonds is discussed in the literature $[2,8,11,23]$. Plato et al. [2] concluded that the effect of one or two hydrogen bonds on $g_{x x}$ is simply additive, and a $g_{x x}$ shift of $400 \mathrm{ppm}$ was calculated for each $\mathrm{H}$-bond. Results of density functional theory calculations showed indeed a $\Delta g_{x x}$ of $440 \mathrm{ppm}$ upon formation of one H-bond and a further decrease of $380 \mathrm{ppm}$ for the addition of the second H-bond [8]. Similar values of $\approx 500 \mathrm{ppm}$ for $g_{x x}^{0}-g_{x x}^{1}$ and $\approx 400 \mathrm{ppm}$ for $g_{x x}^{1}-g_{x x}^{2}$ were obtained with integrated computational approaches based on extended Lagrangian molecular dynamics and discrete-continuum solvent models [23]. 
The average difference between $g_{x x}^{0}$ (representing the non-H-bonded nitroxide population) and $g_{x x}^{1}$ (representing the one H-bonded nitroxide population), as experimentally determined in this work, is $465 \mathrm{ppm}$, and the formation of a second $\mathrm{H}$-bond would account for an additional average decrease of the $g_{x x}$ value by 405 ppm, which is in line with most theoretical predictions (Fig. 5; Table 1).

The analysis performed in this work provides experimental evidence for the presence of three distinct populations of nitroxide spin labels attached to a series of protein sites. The distinction among the three populations is uniquely based on the different number of $\mathrm{H}$-bonds formed $(0,1$, or 2$)$.

As shown in Fig. 5c, it is still possible to analyze the complex $g_{x x}$ features by using a simple single set of average $g$-tensor parameters for the fitting. This would avoid unnecessary complications for those applications, which do not require such a detailed description of the H-bonding properties. In such cases the average $g_{x x}$ values can be plotted versus $A_{z z}$, and the nitroxide micro-environment can be characterized in terms of its position in this plot with respect to the apolar, aprotic region (upper left in Fig. 5c) and the polar, protic region (bottom right in Fig. 5c).

Acknowledgments This work was supported with financial aid of the Deutsche Forschungsgemeinschaft (DFG), projects STE 640/6-2, EN 87/14-2, MO 132/19-2, and the Netherlands Organization for Scientific Research (NWO), project DN 63-248.

Open Access This article is distributed under the terms of the Creative Commons Attribution Noncommercial License which permits any noncommercial use, distribution, and reproduction in any medium, provided the original author(s) and source are credited.

\section{References}

1. E. Bordignon, H.J. Steinhoff, in ESR Spectroscopy in Membrane Biophysics, vol. 27, ed. by M.A. Hemminga, L.J. Berliner (Springer, New York, 2007), pp. 129-164

2. M. Plato, H.J. Steinhoff, C. Wegener, J.T. Törring, A. Savitsky, K. Möbius, Mol. Phys. 100, 37113721 (2002)

3. T.I. Smirnova, T.G. Chadwick, M.A. Voinov, O. Poluektov, J. van Tol, A. Ozarowski, G. Schaaf, M.M. Ryan, V.A. Bankaitis, Biophys. J. 92, 3686-3695 (2007)

4. K. Möbius, A. Savitsky, C. Wegener, M. Plato, M. Fuchs, A. Schnegg, A.A. Dubinskii, Y.A. Grishin, I.A. Grigor'ev, M. Kühn, D. Duché, H. Zimmermann, H.J. Steinhoff, Magn. Reson. Chem. 43, S4S19 (2005)

5. H. Brutlach, E. Bordignon, L. Urban, J.P. Klare, H.-J. Reyer, M. Engelhard, H.J. Steinhoff, Appl. Magn. Reson. 30, 359-372 (2006)

6. D. Kurad, G. Jeschke, D. Marsh, Biophys. J. 85, 1025-1033 (2003)

7. H.J. Steinhoff, A. Savitsky, C. Wegener, M. Pfeiffer, M. Plato, K. Möbius, BBA Bioenergetics 1457, 253-262 (2000)

8. R. Owenius, M. Engström, M. Lindgren, M. Huber, J. Phys. Chem. A 105, 10967-10977 (2001)

9. M. Pavone, A. Sillanpaa, P. Cimino, O. Crescenzi, V. Barone, J. Phys. Chem. B 110, 16189-16192 (2006)

10. A. Savitsky, A.A. Dubinskii, M. Plato, Y.A. Grishin, H. Zimmermann, K. Möbius, J. Phys. Chem. B 112, 9079-9090 (2008)

11. M. Engström, R. Owenius, O. Vahtras, Chem. Phys. Lett. 338, 407-413 (2001)

12. W.R. Hagen, in Advanced EPR, ed. by A.J. Hoff (Elsevier, Amsterdam, 1989), pp. 785-812

13. T.I. Smirnova, A.I. Smirnov, S.V. Paschenko, O.G. Poluektov, J. Am. Chem. Soc. 129, 3476-3477 (2007) 
14. M.G. Finiguerra, H. Blok, M. Ubbink, M. Huber, J. Magn. Reson. 180, 197-202 (2006)

15. O. Burghaus, M. Rohrer, T. Götzinger, M. Plato, K. Möbius, Meas. Sci. Technol. 3, 765-774 (1992)

16. H. Blok, J.A. Disselhorst, S.B. Orlinskii, J. Schmidt, J. Magn. Reson. 166, 92-99 (2004)

17. M. Fuchs, T. Prisner, K. Möbius, Rev. Sci. Instrum. 70, 3681-3683 (1999)

18. H.J. Steinhoff, N. Radzwill, W. Thevis, V. Lenz, D. Brandenburg, A. Antson, G. Dodson, A. Wollmer, Biophys. J. 73, 3287-3298 (1997)

19. L.J. Berliner, J. Grunwald, H.O. Hankovszky, K. Hideg, Anal. Biochem. 119, 450-455 (1982)

20. P.V.L. Padmavathi, H.-J. Steinhoff, J. Mol. Biol. 378, 204-214 (2008)

21. A.A. Wegener, J.P. Klare, M. Engelhard, H.J. Steinhoff, EMBO J. 20, 5312-5319 (2001)

22. M. Pfeiffer, T. Rink, K. Gerwert, D. Oesterhelt, H.J. Steinhoff, J. Mol. Biol. 287, 163-171 (1999)

23. M. Pavone, P. Cimino, O. Crescenzi, A. Sillanpaa, V. Barone, J. Phys. Chem. B 111, 8928-8939 (2007) 